\title{
Ground States of Magnetorheological Fluids
}

\author{
Lei Zhou,* Weijia Wen, and Ping Sheng \\ Department of Physics, The Hong Kong University of Science and Technology, \\ Clear Water Bay, Kowloon, Hong Kong, China
}

(Received 24 March 1998)

\begin{abstract}
Through energy minimization and stability argument, we find the ground states of the magnetorheological fluids to consist of magnetic particles arranged in a body centered tetragonal structure inside spheroidal clusters. The width $D$ of the clusters varies with the length $L$ as $D \sim L^{n}$, where $n=0.62$ for magnetically saturated particles. In the case of magnetically unsaturated particles, the power law variation is divided into two segments, with $n=0.4$ in the first segment and $n=0.62$ in the large $L$ limit. Our results offer a theoretical framework for the resolution of conflicting experimental observations, with the demagnetization field being the crucial physical consideration. [S0031-9007(98)06941-5]
\end{abstract}

PACS numbers: 75.50.Mm, 47.54. $+\mathrm{r}, 82.70 . \mathrm{Kj}$

Magnetorheological (MR) fluids, generally consisting of small magnetic particles dispersed in a liquid, are material systems whose rheological properties are controllable through the application of an external magnetic field. Under a high magnetic field, the magnetic particles have been observed to aggregate into elongated clusters aligned along the magnetic field direction [1-5]. This macrostructure is responsible for the solidlike rheological characteristics and is hereby denoted the ground state of the MR fluids at the high field limit. The structure of the MR fluid ground state(s) has been the subject of prior experimental and theoretical studies, but with conflicting conclusions in regard to both the observations and the governing physics.

In this work, we consider a dipolar model of the MR fluids and use energy minimization and stability argument to derive its high-field ground state(s). The dipolar model can be a reasonably accurate description of those physical systems where the solid particles have a magnetic core and a nonmagnetic outer coating; since the importance of higher multipole interactions varies logarithmically as a function of the surface separation between the particles [6], even a thin nonmagnetic coating, on the order of a few percent of the sphere radius, would limit the importance of multipole interactions in these systems. Through both theoretical calculation and experiments, we show that the structure of the ground state consists of spheroidal clusters, aligned along the field direction, inside which the magnetic particles are arranged in a body-centeredtetragonal (bct) lattice. The width $D$ of the cluster varies with the length $L$ [7] as $D \sim L^{n}$, where the value of $n$ can be different for magnetically saturated particles and magnetically unsaturated particles. In prior experimental works there have indeed been reports of conflicting values of $n \sim 0.67$ [1,2] and $n \sim 0.37$ [5] observed in different systems, with evidence showing a transition between two regimes also reported [3]. Our calculations show that for magnetically saturated and unsaturated particles, $n \sim$ 0.62 and $n \sim 0.4$, respectively, with the unsaturated case exhibiting a second segment of $n \sim 0.62$ in the limit of large $L$. These values are noted to be very close to the two reported ones. Our results thus offer a potential framework for reconciling the conflicting observations, with the demagnetization field being the key element in our physical considerations.

Consider the energy of the magnetic particles system, given by

$$
\begin{aligned}
H= & \frac{1}{2} \sum_{i, j}\left[\frac{\vec{\mu}_{i} \cdot \vec{\mu}_{j}}{r_{i j}^{3}}-\frac{3\left(\vec{\mu}_{i} \cdot \vec{r}_{i j}\right)\left(\vec{\mu}_{j} \cdot \vec{r}_{i j}\right)}{r_{i j}^{5}}\right] \\
& -\vec{h}_{0} \cdot \sum_{i} \vec{\mu}_{i} .
\end{aligned}
$$

Here $\vec{\mu}_{i}$ is the magnetic moment of the $i$ th particle. We wish to consider the two limiting cases of saturated and unsaturated magnetic particles, with the aim of bounding the experimental behaviors. For the saturated case, which will be the first case considered below, $\vec{\mu}_{i}$ is independent of the external field; $\vec{r}_{i j}=\vec{r}_{j}-\vec{r}_{i}$, where $\vec{r}_{i}$ denotes the position of the $i$ th particle; and $\vec{h}_{0}$ is the applied field, directed along the $z$ direction. Ground-state structure is sensitive to the magnetostatic conditions imposed at the cell boundary. In the case of electrorheological (ER) fluids, metallic cell boundaries imply minimal depolarization effect. In the absence of metalliclike boundary conditions, which is generally the case in MR fluid systems, demagnetization field consideration becomes important in determining the structure of the ground state. Figure 1 shows that in a cylindrical-shaped cluster with the axis aligned along the field direction, the calculated demagnetization field $\vec{h}_{d}$ has a nonuniform $z$-component in the corner region of the cluster, leading to a net repulsive force $\vec{F}_{i}=\left|\vec{\mu}_{i}\right|\left(\nabla h_{d}^{z}\right)$ on the particles away from the cluster. Thus the cylindrical shape is not stable. The removal of the particles in the corner regions directly leads to the prolate spheroidal shape [8] as the stable one, in 


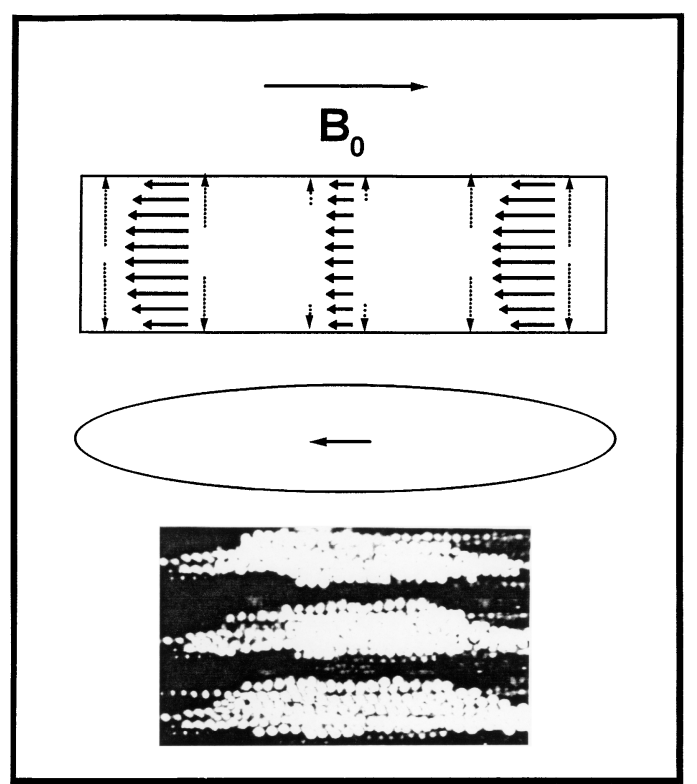

FIG. 1. Schematic pictures depicting the demagnetization fields inside a cylindrical cluster (top) and a prolate spheroidal cluster (middle). The net field inside the cluster is the sum of the externally applied field plus the demagnetization field. Inside the cylindrical cluster, there is a large gradient of the demagnetization field in the corner region, leading to a repulsive force away from the cluster, as indicated by the arrows. The bottom panel shows a picture of three aggregated clusters observed experimentally under an applied magnetic field of $50 \mathrm{G}$, consisting of magnetic particles as described in the text.

which the field is uniform. This is well confirmed experimentally [9], as shown by the picture in Fig. 1 for aggregated magnetic clusters consisting of $36 \mu \mathrm{m}$ diameter $\mathrm{Ni}$-coated glass spheres with an additional $3 \mu \mathrm{m}$ coating of $\mathrm{TiO}_{2}$. Each individual particle is known to possess a small permanent moment.

We next consider a collection of prolate spheroidal clusters with length $L$ and minor-axis diameter $D$ with their centers of mass arranged in a triangular lattice in the $x-y$ plane [10]. The internal structure of the magnetic particles and the value of $D$ as a function of $L$ are the questions to be addressed. The energy of an isolated cluster can be calculated exactly according to Eq. (1). The calculation of Tao and Sun [11] has shown that the bct structure is the lowest in energy for dipoles in an external field, with fcc structure a close second. Thus we consider only these two structures for the particles inside each cluster. The repulsive interaction energy between the clusters is calculated by dividing clusters' centers of mass in the $x-y$ plane into two groups: those inside a circle of large radius $R$ centered on a particular cluster, and those outside the circle. The value of $R$ is chosen so that the final results are insensitive to its further increase. The repulsive energy, calculated on the per-particle basis for the center cluster, would then consist of the contribution from those clusters inside the circle, which is calculated exactly, plus the contribution from those clusters outside the circle, which is accounted for by using the effective medium model as proposed by Grasselli et al. [2]. The latter has the following approximate analytical form:

$$
u_{>} \cong \frac{4 \pi \mu^{2}}{\nu_{p}} \phi\left[\sqrt{1+\left(\frac{R}{L}\right)^{2}}-\frac{R}{L}\right],
$$

where $\nu_{p}$ is the particle volume and $\phi$ the particle volume fraction. In Fig. 2, we show the calculated per particle energies for $L=50 d$, where $d$ denotes the particle diameter, plotted as a function of $D$ for the bct and fcc internal structures. Here $\phi=5 \%$ and $R=100 d$. We have assumed all the $\vec{\mu}_{i}$ 's to be aligned in the $z$-direction [12]. It is seen that there is a value of $D$ which minimizes the total energy, for either the bct case or the fcc case. A second minimum, slightly higher in energy, is also visible in both cases. This second minimum is important for systems consisting of small magnetic particles so that finite temperature fluctuations from the ground states could be important. The fact that bct is the preferred internal structure is verified experimentally by freezing the clusters in epoxy, and taking scanning electron microscope pictures of the cuttings in a plane perpendicular to the applied field, shown as the inset in Fig. 2. The square lattice with neighboring spheres shifted by $d / 2$ in the $z$ direction is characteristic of the bet lattice.

The energy minimum is the result of two dominant contributions. In the region of small $D$, the steep decline is due to the lowering of the bulk energy as $D$ increases. However, the price one pays is the increased demagnetization field, which increases the shape-dependent part of the energy. The latter trend dominates in the limit of large

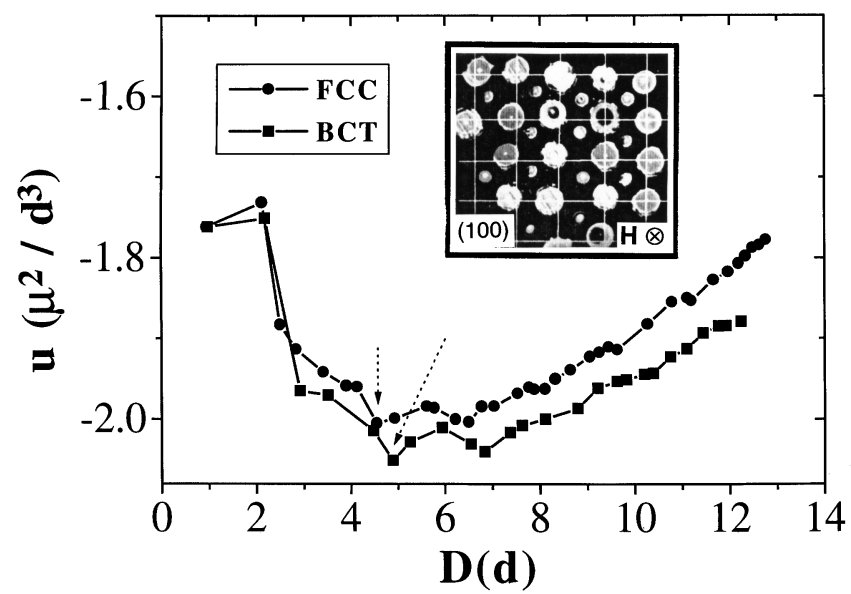

FIG. 2. Calculated energy per particle plotted as a function of the minor axis diameter of the clusters (in units of $d$ ), which are arranged in a two-dimensional triangular lattice. Results for two internal structures, bct and fcc, are shown. The minima are indicated by the arrows. The inset shows the cross-sectional cutting of a cluster frozen in epoxy, giving clear evidence of the bct structure. 
$D$. It follows that the "surface energy" of a cluster, if it were to be defined, would vary as a function of $D$, the position on the cluster surface, as well as the structure internal to the cluster.

In Fig. 3 the ground-state $D-L$ relation thus calculated is plotted as the upper dashed line. The experimental data, shown as solid circles, are for $\phi=5 \%$ of doubly coated particles described above. Good agreement is obtained with no adjustable parameters.

So far we have considered the case of $\vec{\mu}$ being independent from $\vec{h}_{0}$. In the case of unsaturated magnetic particles, we wish to assume the simplest model $\vec{\mu}=\chi \vec{h} \nu_{p}$, where $\vec{h}$ is the local field. This linear dependence between $\vec{\mu}$ and $\vec{h}$ implies weak magnetic fields where the nonlinearity is negligible. Also, it should be noted that to the extent the linear relation holds between $\vec{\mu}$ and $\vec{h}$, the results obtained below for the unsaturated case are independent of the applied field strength. In the approximation where one treats the interior of the cluster as continuous, $\vec{h}$ is given by the sum of the external field and the demagnetization field:

$$
\vec{h}=\vec{h}_{0}-N_{\chi} \vec{h}=\frac{\vec{h}_{0}}{1+N_{\chi}},
$$

with

$$
N=\frac{4 \pi}{q^{2}-1}\left[\frac{q}{\sqrt{q^{2}-1}} \ln \left(q+\sqrt{q^{2}-1}\right)-1\right] \frac{\nu_{p}}{\nu_{c}}
$$

being the demagnetization factor for prolate spheroids, where $q=L / D$, and $\nu_{c}$ is the unit cell volume of the bct structure. By substituting $\chi \vec{h} \nu_{p}$ for $\vec{\mu}$ in Eq. (1), we have calculated the ground-state $D-L$ relation with $\chi=3$. The

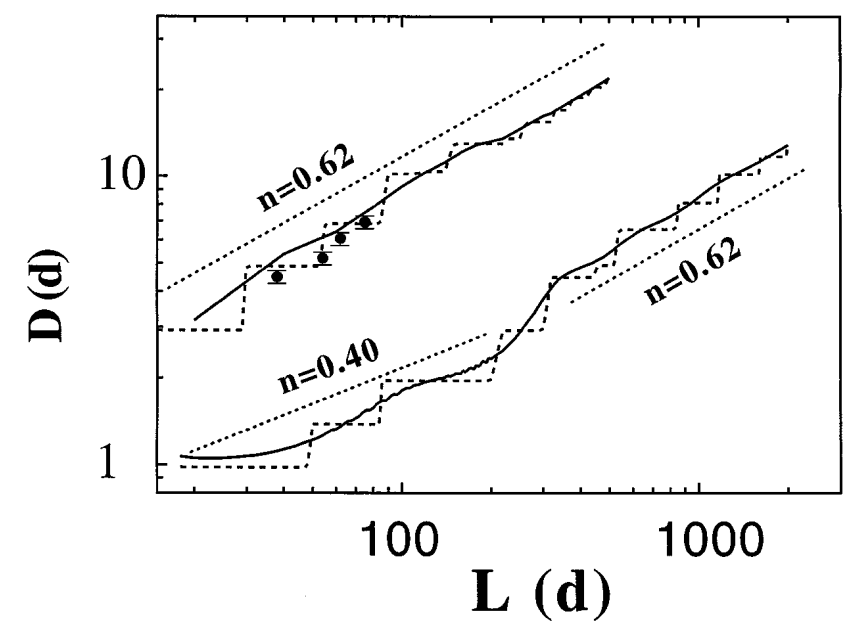

FIG. 3. The calculated $D-L$ relation for the saturated magnetic particles (upper curves) and the unsaturated magnetic particles (lower curves), plotted on the log-log scale. The dashed lines correspond to the lowest energy configurations. The solid lines give the thermally averaged results, with parameters given in the text. The dotted lines are parallel to the best straight-line fits to the solid lines, with the slopes shown. results are shown as the lower dashed line in Fig. 3. The solid lines are the results of finite-temperature calculations with $D=\sum_{i} D_{i} \exp \left(-U_{i} / k T\right) / \sum_{i} \exp \left(-U_{i} / k T\right)$, where $D_{i}$ and $U_{i}$ are the diameter and energy deviation from the ground state of the $i$ th structure, respectively, $k$ is the Boltzmann's constant, and $T$ is the temperature. Here we have used $\left(\mu^{2} \phi S\right) /\left(d^{3} k T\right)=2$ for the saturated case, where $S=\pi D^{2} / 4 d^{2}$, and $\left(\chi^{2} h_{0}^{2} \nu_{p}^{2} \phi S\right) /\left(d^{3} k T\right)=0.3$ for the unsaturated case. These parameter values intend to indicate a temperature high enough as compared to the pairwise dipole interaction energy and implies that the polarizability of the moments is on the same order as $d^{3}$. The effect of thermal averaging is largely to include fluctuations (especially the second minima) so that the $D-L$ relation becomes continuous. That could be the case, for example, if the particles' Brownian motion is nonnegligible, which was not the case for the large doubly coated particles. It is seen that whereas in the saturated case we have $D \sim L^{0.62}$, whereas in the unsaturated case the behavior is divided into two regimes: $D \sim L^{0.4}$ in the small $L$ region, with a transition to $D \sim L^{0.62}$ in the large $L$ limit. The power law behavior is noted to become more exact as $L$ increases. The transition point between the two regimes is controlled by the value of $\chi$ : larger $\chi$ shifts the transition point to larger values of $L$. The physics of the transition between the two regimes is again the demagnetization factor. Since the demagnetization effect is more significant in the small $D-L$ region, it is clear that a small exponent would imply a smaller demagnetization field [13], and hence favor the magnetic energy inside the cluster because of the larger local field [see Eq. (3)]. As the demagnetization effect decreases with increasing $L$, there is no more advantage to be gained by a further decrease in the demagnetization factor, and the exponent reverts to the same as that for the saturated case.

The two calculated cases of saturated and unsaturated magnetic particles define the limiting behaviors for the physical systems. Prior experiments by Grasselli et al. [2] with $0.8 \mu \mathrm{m}$ magnetic particles have shown $D \sim$ $L^{0.67 \pm 0.4}$. The exponent is in reasonable agreement with the large $L$ segment of the unsaturated case. Moreover, at $L \sim 400 d$ the experiment gives $D \sim 15 d$ for the $\phi=5 \%$ sample. These values indeed fall between our unsaturated (large $L$ segment) and the saturated cases. On the other hand, Liu et al. [5] studied ferrofluid emulsions and found $D \sim L^{0.37}$. Also, at $L \sim 100 d$ they found a center-tocenter cluster separation of $\sim 10 d$. By using their value of $\phi=4 \%$, this cluster separation translates into a $D \cong$ 2.4d. Thus both the exponent value and the absolute values of $D$ and $L$ are in excellent agreement with our calculated values in the unsaturated case. Also, in our case the transition point of $200 d$ is in agreement with the fact that starting from $L=100 \mu \mathrm{m}$, the exponent value was found to be increased to $n \approx 0.5$ [5]. However, in their experiment the low exponent region extends to larger $L$ than what we have calculated. This could arise 
if the effective $\chi$ is larger than the value 3 used in our calculation. The transition between the low-exponent and the high-exponent segments is noted to be observed in Ref. [3], with similar exponent values.

In short, we have found the high-field ground state configurations for a system of magnetic particles. The results agree well with those observed experimentally, and serve to resolve conflicting experimental observations and clarify the underlying physics. This work is partially supported by Grant No. SAE95/96.SC01. One of us (L.Z.) is grateful to the Materials Information Science Group of the Institute for Materials Research, Tohoku University, for the supercomputing support.

*Present address: Kawazoe Lab, Institute for Materials Research, Tohoku University, Sendai 980-8577, Japan.

[1] E. Lemaire, Y. Grasselli, and G. Bossis, J. Phys. II (France) 2, 359 (1992).

[2] Y. Grasselli, G. Bossis, and E. Lemaire, J. Phys. II (France) 4, 253 (1994).

[3] H. Wang, Y. Zhu, C. Boyd, W. Luo, A. Cebers, and R. E. Rosensweig, Phys. Rev. Lett. 72, 1929 (1994).

[4] Y.H. Hwang and X. L. Wu, Phys. Rev. E 49, 3102 (1994).

[5] J. Liu, E. M. Lawrence, A. Wu, M. L. Ivey, G. A. Flores, K. Javier, J. Bibette, and J. Richards, Phys. Rev. Lett. 74, 2828 (1995).

[6] In electrostatics, the mutual capacitance of two conducting spheres with radius $R$ and surface-to-surface separation $\delta$ may be easily calculated to be $R \ln (R / \delta)$ in the limit of $R \gg \delta$. This dependence on $\delta$ is responsible for the logarithmic divergence of the dielectric constant of a simple cubic array of nearly touching conducting spheres. See, for example, J. B. Keller, J. Appl. Phys. 34, 991 (1963). The same mathematics applies in the magnetostatic case.

[7] In most cases $L$ is just the cell thickness.

[8] Ellipsoidal shape is stable in general. This fact is a classical result of micromagnetics. In view of the azimuthal symmetry of the system, we consider only the spheroidal case.

[9] It should be noted, however, that for thin clusters the fact that the constituent particles are discrete can mean significant deviations from an idealized ellipsoidal shape. In fact, the optimal shape may resemble a cylinder with two conical-end tips. This could be the case in Ref. [5], where the clusters are thin and cylinderlike. As our calculations are based on summation of interaction energies between discrete particles, these "deviations" from the ellipsoidal shape have been explicitly taken into account in the search of the ground state.

[10] The triangular lattice is chosen for definitiveness and convenience of the calculations. As the repulsive energy is much smaller than the intracluster particle-particle interaction energy, the choice of the cluster lattice structure does not alter any of the results qualitatively.

[11] R. Tao and J. M. Sun, Phys. Rev. Lett. 67, 398 (1991).

[12] This has been checked in a separate calculation, where we have minimized the energy with respect to the magnetic moments' orientations from random starting configurations. The results obtained show that even in zero field, the moments are aligned in the $z$ direction because such alignment is the natural ground state of the system in the bct arrangement.

[13] It should be noted that $L$ is in units of $d$, hence $L>1$ always. 